Mathematical Modelling and Analysis

Volume 7 Number 1, 2002, Pages 117-126

(C) 2002 Technika

\title{
THE MATHEMATICAL MODEL OF COMPRESSIBLE FLUID FLOW ${ }^{1}$
}

\section{T. LEONAVIČIENE ${ }^{1}$ and K. PILECKAS ${ }^{2}$}

Institute of Mathematics and Informatics

Akademijos 4, 2600 Vilnius, Lithuania

E-mail: Terese.Brazauskaite@vpu.1t; pileckas@julius.ktl.mii.lt

Received October 1, 2001

\begin{abstract}
In this note we consider the mathematical model of the isothermal compressible fluid flow in an exterior domain $\Omega \subset \mathbb{R}^{3}$. In order to solve this problem we apply a decomposition scheme and reduce the nonlinear problem to an operator equation with a contraction operator. After the decomposition the nonlinear problem splits into three linear problems: Neumann-like problem, modified Stokes problem and transport equation. These linear problems are solved in weighted function spaces with detached asymptotics.
\end{abstract}

\section{INTRODUCTION}

In this note we study the asymptotic behaviour of steady solutions to equations describing an isothermal motion of compressible viscous fluid in an exterior domain $\Omega \subset \mathbb{R}^{3}$, i.e. $\Omega$ is an open set, exterior to a compact set $B \subset \mathbb{R}^{3}$ with a sufficiently smooth boundary $\partial \Omega$. Moreover, we suppose that the interior of $B$ is non-empty and contains the origin of coordinates. In $\Omega$ we consider the classical Poisson-Stokes equations for unknown functions $\rho$ (density) and $\mathbf{v}=\left(v_{1}, v_{2}, v_{3}\right)$ (velocity):

$$
\left\{\begin{array}{l}
-\mu_{1} \Delta \mathbf{v}-\left(\mu_{1}+\mu_{2}\right) \nabla \operatorname{div} \mathbf{v}+\nabla \rho=\rho \mathbf{b}-\rho(\mathbf{v} \cdot \nabla) \mathbf{v}, \quad x \in \Omega \\
\operatorname{div}(\rho \mathbf{v})=0, \quad x \in \Omega \\
\mathbf{v}=0, \quad x \in \partial \Omega
\end{array}\right.
$$

Here $\mu_{1}, \mu_{2}$ are constant coefficients of shear and bulk viscosities satisfying the conditions

$$
\mu_{1}>0, \quad \mu_{2} \geq-\frac{2}{3} \mu_{1}
$$

\footnotetext{
${ }^{1}$ This work was supported by Lithuanian State Science and Foundation grant A-524.
} 
and $\mathbf{b}$ is a density of external forces. We assume that $\mathbf{b}$ is a "small" perturbation of a "large" potential force, i.e. that $\mathbf{b}$ has the form

$$
\mathbf{b}=\nabla \Phi+\mathbf{f},
$$

where $\Phi$ is a potential which can be (as well as its derivatives) arbitrary "large" and $\mathbf{f}$ is a "small" perturbation of $\nabla \Phi$.

Since the flow domain $\Omega$ is unbounded, the equations (1.1) are supplied with the conditions at infinity. We assume that the velocity field $\mathbf{v}$ tends at infinity to zero and the density $\rho$ to a constant density and prescribe the following conditions

$$
\mathbf{v}(x) \rightarrow 0, \quad \rho(x) \rightarrow \rho_{*}, \quad \rho_{*}=\text { const }>0, \quad|x| \rightarrow \infty .
$$

The exact solution $\left(\rho_{0}, \mathbf{v}_{0}\right)$ of problem (1.1), (1.4) corresponding to the potential force $\nabla \Phi$ (i.e. $\mathbf{f}=0$ ) is the rest state $\left(\rho_{0}, 0\right)$, where $\rho_{0}$ satisfies the equation

$$
\nabla \rho_{0}=\rho_{0} \nabla \Phi .
$$

If $\Phi(x) \rightarrow 0$ as $|x| \rightarrow \infty$, from (1.4), (1.5) we find $\rho_{0}(x)=\rho_{*} \exp \Phi(x)$. The equations for the perturbation $\left(\sigma=\rho-\rho_{0}, \mathbf{v}\right)$ have the form

$$
\left\{\begin{array}{l}
-\mu_{1} \Delta \mathbf{v}-\left(\mu_{1}+\mu_{2}\right) \nabla \operatorname{div} \mathbf{v}+\nabla \sigma-\sigma \nabla \Phi=\mathbf{F}(\sigma, \mathbf{v}), \quad x \in \Omega \\
\operatorname{div}\left(\rho_{0} \mathbf{v}\right)=-\operatorname{div}(\sigma \mathbf{v}), \quad x \in \Omega \\
\mathbf{v}=0, \quad x \in \partial \Omega, \\
\mathbf{v}(x) \rightarrow 0, \quad \sigma(x) \rightarrow 0, \quad|x| \rightarrow \infty
\end{array}\right.
$$

where

$$
F(\sigma, \mathbf{v})=-\left(\rho_{0}+\sigma\right)(\mathbf{v} \cdot \nabla) \mathbf{v}+\left(\rho_{0}+\sigma\right) \mathbf{f} .
$$

A possible linearization of the system (1.6) near the equilibrium state $\left(\rho_{0}, 0\right)$ reads

$$
\left\{\begin{array}{l}
-\mu_{1} \Delta \mathbf{v}-\left(\mu_{1}+\mu_{2}\right) \nabla \operatorname{div} \mathbf{v}+\nabla \sigma-\sigma \nabla \Phi=\mathbf{F}, \quad x \in \Omega \\
\operatorname{div}\left(\rho_{0} \mathbf{v}\right)=-\operatorname{div}(\sigma \mathbf{w}), \quad x \in \Omega, \\
\mathbf{v}=0, \quad x \in \partial \Omega, \quad \mathbf{v}(x) \rightarrow 0, \quad \sigma(x) \rightarrow 0, \quad|x| \rightarrow \infty
\end{array}\right.
$$

where $\sigma, \mathbf{v}$ are unknown, while $\mathbf{F}, \mathbf{w}$ are given.

The solvability of problem (1.1) - (1.4) was studied in [11] using a different decomposition scheme and the techniques due to Matsumura, Nishida [1]. Moreover, in [11] were obtained decay estimates for the solution $\left(\rho=\rho_{0}+\sigma, \mathbf{v}\right)$ of (1.1) - (1.4). In particular, it is shown that the solution is "physically reasonable", i.e. $\mathbf{v}(x)=O\left(|x|^{-1}\right), \quad \nabla \mathbf{v}(x)=O\left(|x|^{-2}\right), \quad \sigma(x)=O\left(|x|^{-2}\right)$. However, the analysis of [11], based on the integral representation formula for the Stokes problem and estimates of weakly singular integrals appearing in 
this representation, does not provide optimal decay rates for the higher order derivatives of the solution. Furthermore, with this method one cannot specify the asymptotic behaviour of the solution as $|x| \rightarrow \infty$.

In this note in order to investigate the solution of problem (1.1) - (1.4) we employ methods related with the application of weighted function spaces (e.g. $[2 ; 8])$. However, already the Navier-Stokes equations of the incompressible fluid motion are not solvable in classical weighted Sobolev (Hölder) spaces while considering the convective term $(\mathbf{v} \cdot \nabla) \mathbf{v}$ as a perturbation of the Stokes problem. On the other hand, if we study these equations in function spaces which elements take suitable asymptotic forms (i.e. the elements are defined as a sum of two parts one of which contains the main asymptotic term and another one belongs to the usual weighted space), then the NavierStokes problem is well-posed (see $[6 ; 7])$. We also refer to [3], [4; 5; 10] where such weighted spaces with detached asymptotics were successfully applied to study Navier-Stokes equations in other unbounded domains and to [9] were the spaces with detached asymptotic were applied to study viscoelastic flows in an exterior domain.

In order to solve the linearized problem (1.8) and the nonlinear problem (1.1), we apply the decomposition scheme proposed in [12] (see also the references in [12] for the original application of the similar decomposition scheme to steady compressible Navier-Stokes equations linearized on a constant density).

In this note we present only the general scheme and the main idea of the proof for the solvability of problem (1.1) (the complete proofs will be published separatly).

The authors are grateful to Professor S.A. Nazarov for the useful discussions.

\section{DECOMPOSITION SCHEME}

We will consider a slightly more general than (1.8) system

$$
\left\{\begin{array}{l}
-\mu_{1} \Delta \mathbf{v}-\left(\mu_{1}+\mu_{2}\right) \nabla \operatorname{div} \mathbf{v}+\nabla \sigma-\sigma \nabla \Phi=\mathbf{F}, \quad x \in \Omega \\
\operatorname{div}\left(\rho_{0} \mathbf{v}\right)=-\operatorname{div}(\sigma \mathbf{w})+g, \quad x \in \Omega, \\
\mathbf{v}=0, \quad x \in \partial \Omega, \quad \mathbf{v}(x) \rightarrow 0, \quad \sigma(x) \rightarrow 0, \quad|x| \rightarrow \infty
\end{array}\right.
$$

where $g$ is given.

We represent the velocity field $\mathbf{v}$ as a sum $\mathbf{v}=\mathbf{u}+\nabla \varphi$, where the functions $u$ and $\varphi$ satisfy the conditions $\operatorname{div}\left(\rho_{0} \mathbf{u}\right)=0, x \in \Omega ; \quad \mathbf{u} \cdot \mathbf{n}=0, x \in \partial \Omega$, and $\frac{\partial \varphi}{\partial n}=0, \quad x \in \partial \Omega$. Then the system (2.1) splits into the following three simpler problems:

$$
\left\{\begin{array}{l}
\Delta_{\rho_{0}} \varphi=-\operatorname{div}(\sigma \mathbf{w})+g, \quad x \in \Omega \\
\frac{\partial \varphi}{\partial n}=0, \quad x \in \partial \Omega, \quad \varphi(x) \rightarrow 0, \quad|x| \rightarrow \infty
\end{array}\right.
$$




$$
\begin{aligned}
& \left\{\begin{array}{l}
-\mu_{1} \Delta \mathbf{u}-\left(\mu_{1}+\mu_{2}\right) \nabla \operatorname{div} \mathbf{u}+\rho_{0} \nabla\left(\Pi / \rho_{0}\right)=\mathbf{G}, \quad x \in \Omega, \\
\operatorname{div}\left(\rho_{0} \mathbf{u}\right)=0, \quad x \in \Omega, \\
\mathbf{u}=-\nabla \varphi, \quad x \in \partial \Omega, \\
\mathbf{u}(x) \rightarrow 0, \quad \Pi(x) \rightarrow 0, \quad|x| \rightarrow \infty,
\end{array}\right. \\
& \sigma+\left(2 \mu_{1}+\mu_{2}\right) \operatorname{div}\left(\frac{\sigma \mathbf{w}}{\rho_{0}}\right)=\Pi, \quad x \in \Omega,
\end{aligned}
$$

where

$$
\begin{aligned}
\mathbf{G}=\mathbf{F} & +\left(2 \mu_{1}+\mu_{2}\right) \nabla\left(\rho_{0}^{-2} \nabla \rho_{0} \cdot \mathbf{w} \sigma\right)-\left(2 \mu_{1}+\mu_{2}\right) \nabla\left(\rho_{0}^{-1} \nabla \rho_{0} \cdot \nabla \varphi\right) \\
& -\left(2 \mu_{1}+\mu_{2}\right) \rho_{0}^{-1} \nabla \rho_{0} \operatorname{div}\left(\frac{\sigma \mathbf{w}}{\rho_{0}}\right)+\left(2 \mu_{1}+\mu_{2}\right) \nabla\left(\frac{g}{\rho_{0}}\right) .
\end{aligned}
$$

The solution $(\sigma, \mathbf{v})$ of the problem (2.1) can be found as a fixed point of the linear mapping

$$
\mathcal{L}: \tau \longmapsto \sigma
$$

which we define in the following way:

(a) for given $\tau$ we find $\varphi$ by solving the Neumann problem (2.2);

(b) next we find the solution $(\mathbf{u}, \Pi)$ of the Stokes-type problem (2.3) using (2.5) with $\sigma=\tau$;

(c) finally, we find $\sigma$ from the transport equation (2.4).

\section{SOLVABILITY OF THE AUXILIARY PROBLEMS}

\subsection{Function spaces}

- $C^{\infty}(\Omega)$ is the set of all infinitely differentiable in $\bar{\Omega}$ functions; $C_{0}^{\infty}(\Omega)$ is the subset of functions from $C^{\infty}(\Omega)$ having compact supports in $\Omega ; C_{0}^{\infty}(\bar{\Omega})$ is the set of functions from $C^{\infty}(\Omega)$ which are equal to zero in the neighborhood of infinity, i.e. for sufficiently large $|x|$ (but not necessary on $\partial \Omega$ ).

- $W^{l, q}(\Omega), l \geq 0, q \in(1, \infty)$, is the usual Sobolev space and $\stackrel{\circ}{W}^{l, q}(\Omega)$ is the closure of $C_{0}^{\infty}(\Omega)$ in the norm $\left\|\cdot ; W^{l, q}(\Omega)\right\| ; L^{q}(\Omega)=W^{0, q}(\Omega)$.

- Let $\mathbb{S}^{2}$ be a unit sphere in $\mathbb{R}^{3}$. $W^{l, q}\left(\mathbb{S}^{2}\right)$ - Sobolev space of functions defined on $\mathbb{S}^{2}$.

- $V_{\beta}^{l, q}(\Omega), l \geq 0, q \in(1, \infty), \beta \in \mathbb{R}$, is the closure of $C_{0}^{\infty}(\bar{\Omega})$ in the weighted norm

$$
\left\|u ; V_{\beta}^{l, q}(\Omega)\right\|=\sum_{|\alpha| \leq l}\left\||x|^{\beta-l+|\alpha|} D^{\alpha} u ; L^{q}(\Omega)\right\|
$$

where $\alpha=\left(\alpha_{1}, \alpha_{2}, \alpha_{3}\right), D^{\alpha}=\partial^{|\alpha|} / \partial x_{1}^{\alpha_{1}} \partial x_{2}^{\alpha_{2}} \partial x_{3}^{\alpha_{3}}, \alpha_{j} \geq 0,|\alpha|=\alpha_{1}+\alpha_{2}+\alpha_{3}$. 
- $\Lambda_{\beta}^{l, \delta}(\Omega)$ is the weighted Hölder space defined as the closure of $C_{0}^{\infty}(\bar{\Omega})$ in the norm

$$
\begin{aligned}
& \left\|u ; \Lambda_{\beta}^{l, \delta}(\Omega)\right\|=\sum_{|\alpha| \leq l} \sup _{x \in \bar{\Omega}}\left(|x|^{\beta-l-\delta+|\alpha|}\left|D^{\alpha} u(x)\right|\right) \\
& +\sum_{|\alpha|=l} \sup _{x \in \Omega}\left\{|x|^{\beta} \sup _{\substack{y \in \Omega \\
|x-y|<|x| / 2}}\left(|x-y|^{-\delta}\left|D_{x}^{\alpha} u(x)-D_{y}^{\alpha} u(y)\right|\right)\right\} \text {. }
\end{aligned}
$$

- $\mathfrak{V}_{\gamma, k}^{(l, s), q}(\Omega), l \geq 0, q \in(1, \infty), s \geq l, k \in \mathbb{Z}, \gamma \in(l-3 / q+k, l+1-3 / q+k)$, is the weighted Sobolev space with detached asymptotics, i.e. the space of functions admiting the asymptotic representation

$$
u(x)=r^{-k} \mathfrak{U}(\theta)+\widetilde{u}(x),
$$

where $r=|x|, \theta \in \mathbb{S}^{2}, \mathfrak{U} \in W^{s, q}\left(\mathbb{S}^{2}\right), \widetilde{u} \in V_{\gamma}^{l, q}(\Omega)$. The norm in $\mathfrak{V}_{\gamma, k}^{(l, s), q}(\Omega)$ is defined by $\left\|u ; \mathfrak{V}_{\gamma, k}^{(l, s), q}(\Omega)\right\|=\left\|\mathfrak{U} ; W^{s, q}\left(\mathbb{S}^{2}\right)\right\|+\left\|\widetilde{u} ; V_{\gamma}^{l, q}(\Omega)\right\|$.

Functions $\mathfrak{U}$ and $\widetilde{u}$ are called the attributes of $u \in \mathfrak{V}_{\gamma, k}^{(l, s), q}(\Omega)$.

\subsection{Neumann problem}

Let us consider the Neumann problem for the operator $\Delta_{\rho_{0}}=\operatorname{div}\left(\rho_{0}(x) \Delta\right)$, where $\rho_{0}(x)=\rho_{*} \exp \Phi(x), \Phi(x) \in \Lambda_{l+1+\delta+\gamma_{0}}^{l+1, \delta}(\Omega), \gamma_{0}>1$ :

$$
\left\{\begin{aligned}
-\Delta_{\rho_{0}} \varphi & =\psi, \quad x \in \Omega, \\
\frac{\partial \varphi}{\partial n} & =0, \quad x \in \partial \Omega, \quad \varphi(x) \rightarrow 0, \quad|x| \rightarrow \infty .
\end{aligned}\right.
$$

Theorem 3.1. Let $\psi \in V_{\gamma}^{l-1, q}(\Omega), l \geq 1, q>1, \gamma \in(l+2-3 / q, l+3-3 / q)$. Then problem (3.2) has a unique solution $\varphi$ which admits the asymptotic representation $\varphi(x)=c_{0} \cdot \frac{1}{2 \pi|x|}+\widetilde{\varphi}(x)$ with $\widetilde{\varphi} \in V_{\gamma}^{l+1, q}(\Omega)$. There holds the estimate

$$
\left\|\widetilde{\varphi} ; V_{\gamma}^{l+1, q}(\Omega)\right\|+\left|c_{0}\right| \leq c\left\|\psi ; V_{\gamma}^{l-1, q}(\Omega)\right\|
$$

The proof of Theorem 3.1 can be found in [8].

\subsection{Stokes and modified "Stokes" problems}

Let us consider first the Stokes problem

$$
\left\{\begin{array}{l}
-\nu \Delta \mathbf{u}+\nabla p=\mathbf{f}, \quad x \in \Omega \\
\operatorname{div} \mathbf{u}=g, \quad x \in \Omega \\
\mathbf{u}=\mathbf{h}, \quad x \in \partial \Omega
\end{array}\right.
$$


We study problem (3.3) in weighted Sobolev spaces with detached asymptotics. Denote

$$
\begin{aligned}
& \mathcal{D}_{\beta}^{l, q} V(\Omega) \equiv V_{\beta}^{l+1, q}(\Omega) \times V_{\beta}^{l, q}(\Omega) \\
& \mathcal{R}_{\beta}^{l, q} V(\Omega) \equiv V_{\beta}^{l-1, q}(\Omega) \times V_{\beta}^{l, q}(\Omega) \times W^{l+1-1 / q, q}(\partial \Omega), \\
& \mathcal{D}_{\gamma}^{l, q} \mathfrak{V}(\Omega) \equiv \mathfrak{V}_{\gamma, 1}^{(l+1, l+2), q}(\Omega) \times \mathfrak{V}_{\gamma, 2}^{l, l+1}(\Omega), \\
& \mathcal{R}_{\gamma}^{l, q} \mathfrak{V}(\Omega) \equiv \mathfrak{V}_{\gamma, 3}^{(l-1, l), q}(\Omega) \times \mathfrak{V}_{\gamma, 2}^{(l, l+1), q}(\Omega) \times W^{l+1-1 / q, q}(\partial \Omega)
\end{aligned}
$$

with $l \geq 1, q \in(1, \infty), \beta \in \mathbb{R}, \gamma \in(l+2-3 / q, l+3-3 / q)$. Let $S_{\beta}^{l, q}: \mathcal{D}_{\beta}^{l, q} V(\Omega) \mapsto$ $\mathcal{R}_{\beta}^{l, q} V(\Omega)$ be the operator of the Stokes problem (3.3). There holds the following statements.

Theorem 3.2. (see $[7 ; 6])$ (i) If $\beta \in(l+1-3 / q, l+2-3 / q)$, then the mapping $(\mathbf{u}, p) \longmapsto(\mathbf{f}, g, \mathbf{h})=S_{\beta}^{l, q}(\mathbf{u}, p)$ is an isomorphism.

(ii) Let $(\mathbf{f}, g, \mathbf{h}) \in \mathcal{R}_{\gamma}^{l, q} V(\Omega) \subset \mathcal{R}_{\beta}^{l, q} V(\Omega)$ with

$$
\gamma \in(l+2-3 / q, l+3-3 / q) .
$$

Then the solution $(\mathbf{u}, p) \in \mathcal{D}_{\beta}^{l, q} V(\Omega)$ admits the asymptotic representation $(\mathbf{u}, p)=\left(\mathbf{u}^{0}, p^{0}\right)+(\widetilde{\mathbf{u}}, \widetilde{p})$, where $(\widetilde{\mathbf{u}}, \widetilde{p}) \in \mathcal{D}_{\gamma}^{l, q} V(\Omega)$ and $\left(\mathbf{u}^{0}, p^{0}\right)=b_{1} \mathbf{E}^{(1)}+$ $b_{2} \mathbf{E}^{(2)}+b_{3} \mathbf{E}^{(3)}$, with $\mathbf{E}^{(j)}$ denoting the $j$-th column of the fundamental matrix for the Stokes operator in $\mathbb{R}^{3}$ and $b_{j} \in \mathbb{R}, j=1,2,3$. Moreover, there holds the estimate

$$
\left\|(\widetilde{\mathbf{u}}, \widetilde{p}) ; \mathcal{D}_{\gamma}^{l, q} V(\Omega)\right\|+\left|b_{1}\right|+\left|b_{2}\right|+\left|b_{3}\right| \leq c\left\|(\mathbf{f}, g, \mathbf{h}) ; \mathcal{R}_{\gamma}^{l, q} V(\Omega)\right\| .
$$

Remark 3.1. The columns of the fundamental matrix for the Stokes operator in $\mathbb{R}^{3}$ are defined by $\mathbf{E}^{(j)}(x)=\frac{1}{8 \pi \nu|x|^{3}}\left(\delta_{j 1}|x|^{2}+x_{1} x_{j}, \delta_{j 2}|x|^{2}+x_{2} x_{j}, \delta_{j 3}|x|^{2}+\right.$ $\left.x_{3} x_{j}, 2 \nu x_{j}\right)^{\top}, j=1,2,3$.

Theorem 3.3. (see $[7 ; 6])$. Let $(\mathbf{f}, g, \mathbf{h}) \in \mathcal{R}_{\gamma}^{l, q} \mathfrak{V}(\Omega)$ with $\gamma$ satisfying (3.4). Then problem (3.3) has a solution $(\mathbf{u}, p) \in \mathcal{D}_{\gamma}^{l, q} \mathfrak{V}(\Omega)$ if and only if there holds the compatibility condition

$$
\int_{\mathbb{S}^{2}} \mathfrak{F}(\theta) d s_{\theta}=0
$$

where $\mathfrak{F}(\theta), \widetilde{\mathbf{f}}$ are the attributes of the function $\mathbf{f}$ in representation (3.1). The solution is unique and there holds the estimate

$$
\left\|(\mathbf{u}, p) ; \mathcal{D}_{\gamma}^{l, q} \mathfrak{V}(\Omega)\right\| \leq c\left\|(\mathbf{f}, g, \mathbf{h}) ; \mathcal{R}_{\gamma}^{l, q} \mathfrak{V}(\Omega)\right\| .
$$


Let us consider now the following "modified Stokes" problem

$$
\left\{\begin{array}{l}
-\mu_{1} \Delta \mathbf{u}-\left(\mu_{1}+\mu_{2}\right) \nabla \operatorname{div} \mathbf{u}+\rho_{0} \nabla\left(\Pi / \rho_{0}\right)=\mathbf{f}, \quad x \in \Omega \\
\operatorname{div}\left(\rho_{0} \mathbf{u}\right)=g, \quad x \in \Omega \\
\mathbf{u}=\mathbf{h}, \quad x \in \partial \Omega
\end{array}\right.
$$

where $\rho_{0}(x)=\rho_{*} \exp (\Phi(x)), \Phi \in \Lambda_{l+1+\delta+\gamma_{0}}^{l+1, \delta}(\Omega), \gamma_{0}>1$.

Theorem 3.4. Let $(\mathbf{f}, g, \mathbf{h}) \in \mathcal{R}_{\gamma}^{l, q} \mathfrak{V}(\Omega), l \geq 1, q \in[6 / 5, \infty), \gamma \in(l+2-$ $3 / q, l+3-3 / q)$ and let $\mathfrak{F}(\theta)$ satisfies the compatibility condition $\int_{\mathbb{S}^{2}} \mathfrak{F}(\theta) d s_{\theta}=0$.

Then problem (3.6) has a unique solution $(\mathbf{u}, \Pi) \in \mathcal{D}_{\gamma}^{l, q} \mathfrak{V}(\Omega)$. There holds the estimate $\left.\left\|(\mathbf{u}, \Pi) ; \mathcal{D}_{\gamma}^{l, q} \mathfrak{V}(\Omega)\right\| \leq c \| \mathbf{f}, g, \mathbf{h}\right) ; \mathcal{R}_{\gamma}^{l, q} \mathfrak{V}(\Omega) \|$.

The proof of Theorem 3.4 consists from several steps. First, we consider the case $\mathbf{h}=0$ and prove the existence of a weak solution $(\mathbf{u}, \Pi) \in V_{0}^{1,2}(\Omega) \times L^{2}(\Omega)$ of the problem (3.6) satisfying the equation $\operatorname{div}\left(\rho_{0} \mathbf{u}\right)=g$ and the integral identity

$$
\begin{aligned}
\mu_{1} \int_{\Omega} \nabla \mathbf{u}: \nabla \boldsymbol{\eta} \mathrm{d} x & +\left(\mu_{1}+\mu_{2}\right) \int_{\Omega} \operatorname{div} \mathbf{u} \operatorname{div} \boldsymbol{\eta} \mathrm{d} x-\int_{\Omega} \rho_{0}^{-1} \Pi \operatorname{div}\left(\rho_{0} \boldsymbol{\eta}\right) \mathrm{d} x \\
& =\int_{\Omega} \mathbf{f} \cdot \boldsymbol{\eta} \mathrm{d} x, \quad \forall \boldsymbol{\eta} \in V_{0}^{1,2}(\Omega)
\end{aligned}
$$

Second, we write (3.6) in the form

$$
\left\{\begin{array}{l}
-\mu_{1} \Delta \mathbf{u}+\nabla \Pi=\mathbf{f}+\rho_{0}^{-1} \nabla \rho_{0} \Pi-\left(\mu_{1}+\mu_{2}\right) \nabla\left(\rho_{0}^{-1} \nabla \rho_{0} \cdot \mathbf{u}\right), \quad x \in \Omega \\
\operatorname{div} \mathbf{u}=g-\rho_{0}^{-1} \nabla \rho_{0} \cdot \mathbf{u}, \quad x \in \Omega \\
\mathbf{u}=\mathbf{h}, \quad x \in \partial \Omega
\end{array}\right.
$$

and consider $(\mathbf{u}, \Pi) \in V_{0}^{1,2}(\Omega) \times L^{2}(\Omega)$ as a solution of the Stokes problem (3.3) with the right-hand side

$$
\begin{aligned}
& \mathbf{f}_{1}=\mathbf{f}+\Pi \nabla \Phi-\left(\mu_{1}+\mu_{2}\right) \nabla(\nabla \Phi \cdot \mathbf{u}) \\
& g_{1}=g-\nabla \Phi \cdot \mathbf{u} \\
& \mathbf{h}=0
\end{aligned}
$$

(remember that $\left.\nabla \Phi=\rho_{0}^{-1} \nabla \rho_{0}\right)$. Using results on the Stokes problem (see Theorems 3.2, 3.3) and bootstrapping arguments we prove Theorem 3.4 in the case of homogeneous boundary conditions (i.e., when $\mathbf{h}=0$ ). Note that bootstraping arguments are based on the following embedding results for weighted function spaces: 
Lemma 3.1. Let $u \in V_{\beta}^{l, q}(\Omega)$.

(i) If $q l \leq 3$ with $q \leq s \leq 3 q /(3-q l)$, then $u \in V_{\beta-l-3 / s+3 / q}^{0, s}(\Omega)$ and $\left\|u ; V_{\beta-l-3 / s+3 / q}^{0, s}(\Omega)\right\| \leq c\left\|u ; V_{\beta}^{l, q}(\Omega)\right\|$.

(ii) If $q l>3$ and $m+\delta \leq l-3 / q$ with $\delta \in(0,1)$, then $u \in \Lambda_{m+\delta+\beta-l+3 / q}^{m, \delta}(\Omega)$ and $\left\|u ; \Lambda_{m+\delta+\beta-l+3 / q}^{m, \delta}(\Omega)\right\| \leq c\left\|u ; V_{\beta}^{l, q}(\Omega)\right\|$.

Lemma 3.2. Let $v \in \Lambda_{l+1+\delta+\beta_{1}}^{l+1, \delta}(\Omega)$.

(i) If $u \in V_{\beta_{2}}^{m, q}(\Omega), m \leq l+1$, then $v u \in V_{\beta_{1}+\beta_{2}}^{m, q}(\Omega)$ and $\left\|v u ; V_{\beta_{1}+\beta_{2}}^{m, q}(\Omega)\right\| \leq$ $c\left\|v ; \Lambda_{l+1+\delta+\beta_{1}}^{l+1, \delta}(\Omega)\right\|\left\|u ; V_{\beta_{2}}^{m, q}(\Omega)\right\|$.

(ii) If $u \in \Lambda_{\beta_{2}}^{m, \delta}(\Omega), m \leq l+1$, then $v u \in \Lambda_{\beta_{1}+\beta_{2}}^{m, \delta}(\Omega)$ and

$$
\left\|v u ; \Lambda_{\beta_{1}+\beta_{2}}^{m, \delta}(\Omega)\right\| \leq c\left\|v ; \Lambda_{l+1+\delta+\beta_{1}}^{l+1, \delta}(\Omega)\right\|\left\|u ; \Lambda_{\beta_{2}}^{m, \delta}(\Omega)\right\| .
$$

Finally, the problem (3.6) with nonhomogeneous boundary conditions is reduced to one with $\mathbf{h}=0$ by using the extension operator.

\subsection{Transport equation}

Let us consider the transport equation

$$
z+\operatorname{div}(\mathbf{w} z)=h, \quad x \in \Omega,
$$

where $\mathbf{w}$ satisfies the condition

$$
\mathbf{w} \cdot \mathbf{n}=0, \quad x \in \partial \Omega .
$$

Here we need results concerning the solvability of (3.8), (3.9) in weighted Sobolev spaces with detached asymptotics.

Theorem 3.5. Let $h \in \mathfrak{V}_{\gamma, 2}^{(l, l+1), q}(\Omega)$ with $l \geq 2, q>3 / 2, \gamma \in(l+2-3 / q, l+$ $3-3 / q)$ and let $\mathbf{w} \in \mathfrak{V}_{\gamma, 1}^{(l+1, l+2), q}(\Omega)$. There exists a number $\varepsilon_{0}>0$ such that if

$$
\left\|\mathbf{w} ; \mathfrak{V}_{\gamma, 1}^{(l+1, l+2), q}(\Omega)\right\| \leq \varepsilon_{0}
$$

then problem (3.8), (3.9) has just one solution $z$ with $z \in \mathfrak{V}_{\gamma, 2}^{(l, l+1), q}(\Omega)$, $\operatorname{div}(\mathbf{w} z) \in V_{\gamma}^{l, q}(\Omega)$. There holds the estimate

$$
\left\|z ; \mathfrak{V}_{\gamma, 2}^{(l, l+1), q}(\Omega)\right\|+\left\|\operatorname{div}(\mathbf{w} z) ; V_{\gamma}^{l, q}(\Omega)\right\| \leq c\left\|h ; \mathfrak{V}_{\gamma, 2}^{(l, l+1), q}(\Omega)\right\| .
$$

Furthermore, $\Delta z \in \mathfrak{V}_{\gamma, 4}^{(l-2, l-1), q}(\Omega), \Delta \operatorname{div}(\mathbf{w} z) \in V_{\gamma}^{l-2, q}(\Omega)$ and

$$
\begin{aligned}
& \left\|\Delta z ; \mathfrak{V}_{\gamma, 4}^{(l-2, l-1), q}(\Omega)\right\|+\left\|\Delta \operatorname{div}(\mathbf{w} z) ; V_{\gamma}^{l-2, q}(\Omega)\right\| \\
& \quad \leq c\left(\left\|h ; \mathfrak{V}_{\gamma, 2}^{(l, l+1), q}(\Omega)\right\|+\left\|\mathbf{w} ; \mathfrak{V}_{\gamma, 1}^{(l+1, l+2), q}(\Omega)\right\|\left\|z ; \mathfrak{V}_{\gamma, 2}^{(l, l+1), q}(\Omega)\right\|\right) .
\end{aligned}
$$




\section{THE SOLVABILITY OF LINEARIZED PROBLEM AND THE NONLINEAR PROBLEM}

Let us consider the linearized problem (2.1).

Theorem 4.1. Let $\Omega \in \mathbb{R}^{3}$ be an exterior domain with the smooth boundary $\partial \Omega, \Phi \in \Lambda_{l+1+\delta+\gamma_{0}}^{l+1, \delta}(\Omega), \gamma_{0}>1, l \geq 2, \delta \in(0,1), \rho_{0}(x)=\rho_{*} \exp \Phi(x)$. Suppose that $\mathbf{w} \in \mathfrak{V}_{\gamma, 1}^{(l+1, l+2), q}(\Omega), \mathbf{F} \in \mathfrak{V}_{\gamma, 3}^{(l-1, l), q}(\Omega), g \in V_{\gamma}^{l, q}(\Omega), q>3 / 2, \gamma \in$ $(l+2-3 / q, l+3-3 / q)$, be given functions with $\mathbf{w}=0, \quad \operatorname{div} \mathbf{w}=0, \quad x \in \partial \Omega$, and $\mathbf{F}$ satisfies the compatibility condition (3.5). There exists a number $\varepsilon_{0}>0$ such that if $\left\|\mathbf{w} ; \mathfrak{V}_{\gamma, 1}^{(l+1, l+2), q}(\Omega)\right\|<\varepsilon_{0}$, then problem $(2.1)$ has a unique solution $(\sigma, \mathbf{v}) \in \mathfrak{V}_{\gamma, 2}^{(l, l+1), q}(\Omega) \times \mathfrak{V}_{\gamma, 1}^{(l+1, l+2), q}(\Omega)$. Moreover, $\operatorname{div}(\mathbf{w} \sigma) \in V_{\gamma}^{l+1, q}(\Omega)$ and there hold the estimates

$$
\begin{aligned}
\left\|\sigma ; \mathfrak{V}_{\gamma, 2}^{(l, l+1), q}(\Omega)\right\|+ & \left\|\mathbf{v} ; \mathfrak{V}_{\gamma, 1}^{(l+1, l+2), q}(\Omega)\right\| \\
& \leq c\left(\left\|\mathbf{F} ; \mathfrak{V}_{\gamma, 3}^{(l-1, l)}, q(\Omega)\right\|+\left\|g ; V_{\gamma}^{l, q}(\Omega)\right\|\right) \\
\left\|\operatorname{div}(\mathbf{w} \sigma) ; V_{\gamma}^{l, q}(\Omega)\right\| & \leq c\left(\left\|\mathbf{F} ; \mathfrak{V}_{\gamma, 3}^{(l-1, l), q}(\Omega)\right\|+\left\|g ; V_{\gamma}^{l, q}(\Omega)\right\|\right)
\end{aligned}
$$

The solvability of (2.1) is proved using the linear mapping $\mathcal{L}: \tau \longmapsto \sigma$ defined by (2.6). If $\varepsilon_{0}$ is small enough, we prove that the mapping $\mathcal{L}$ is a contraction and, therefore, $\mathcal{L}$ has a fixed point $\sigma$. Taking in (2.2)-(2.5) $\tau=\sigma$ we find $\varphi$ - the solution of Neumann problem $(2.2)$ and $(\mathbf{u}, \Pi)$ - the solution of modified Stokes problem (2.3). The solution $(\sigma, \mathbf{v})$ of problem $(2.1)$ is then $(\sigma, \mathbf{v}=\mathbf{u}+\nabla \varphi)$.

While linearized system (1.8) is solved, we find the solution of the nonlinear problem (1.6), (1.7) in the form $(\sigma, \mathbf{v}=\nabla \varphi+\mathbf{u})$, where $(\sigma, \varphi, \mathbf{u})$ is a fixed point of the nonlinear mapping $\mathcal{N}:(\tau, \xi, \mathbf{z}) \rightarrow(\sigma, \varphi, \mathbf{u})$ with $(\sigma, \varphi, \mathbf{u})$ being a solution of the linear problem (1.8) taking in it $\mathbf{F}=\mathbf{F}(\tau, \nabla \xi+\mathbf{z})$ and $\mathbf{w}=\nabla \xi+z$. Using the form $\left(\rho_{0}+\sigma\right)(\mathbf{v} \cdot \nabla) \mathbf{v}$ of the convective term we prove that $\left(\rho_{0}+\tau\right)(\mathbf{w} \cdot \nabla) \mathbf{w}$ satisfies the compatibility condition (3.5) for every $\tau \in \mathfrak{V}_{\gamma, 2}^{(l, l+1), q}(\Omega), \mathbf{w} \in \mathfrak{V}_{\gamma, 1}^{(l+1, l+2), q}(\Omega)$ and, therefore, the nonlinear mapping is defined correctly. Moreover, for small data we prove that this mapping is a contraction in certain small ball of the space $\mathfrak{V}_{\gamma, 2}^{(l, l+1), q}(\Omega) \times \mathfrak{V}_{\gamma, 1}^{(l+1, l+2), q}(\Omega)$ and, hence, it has a unique fixed point - the solution of problem (1.6), (1.7). Finally, we get the main result of the paper.

Theorem 4.2. Let $\Omega \in \mathbb{R}^{3}$ be an exterior domain with the smooth boundary $\partial \Omega, \Phi \in \Lambda_{l+1+\delta+\gamma_{0}}^{l+1, \delta}(\Omega), \mathbf{f} \in \mathfrak{V}_{\gamma, 3}^{(l-1, l), q}(\Omega), l \geq 2, \delta \in(0,1), q>3 / 2, \gamma \in(l+$ $2-3 / q, l+3-3 / q), \gamma_{0}>1$. Suppose that the function $\mathbf{f}$ satisfies compatibility condition (3.5). There exists a number $\varepsilon_{*}>0$ such that if $\left\|\mathbf{f} ; \mathfrak{V}_{\gamma, 3}^{(l-1, l), q}(\Omega)\right\|<$ $\varepsilon_{*}$, then problem $(1.6),(1.7)$ has exactly one solution $(\sigma, \mathbf{v}) \in \mathfrak{V}_{\gamma, 2}^{(l, l+1), q}(\Omega) \times$ 
$\mathfrak{V}_{\gamma, 1}^{(l+1, l+2), q}(\Omega)$ satisfying the estimate $\left\|\sigma ; \mathfrak{V}_{\gamma, 2}^{(l, l+1), q}(\Omega)\right\|+\left\|\mathbf{v} ; \mathfrak{V}_{\gamma, 1}^{(l+1, l+2), q}(\Omega)\right\| \leq$ $c\left\|\mathbf{f} ; \mathfrak{V}_{\gamma, 3}^{(l-1, l), q}(\Omega)\right\|$.

\section{REFERENCES}

[1] A. Matsumura and T. Nishida. Initial boundary value problems for equations of motion of compressible viscous and heat-conductive fluids. Comm. Math. Phys., 89, 445-464, 1998.

[2] S.A. Nazarov. Weighted spaces with detached asymptotics in application to the NavierStokes equations. In: M. Rokyta J. Malek, J Nečas(Ed.), Advances in Math. Fluid Mechanics, Lecture Notes of the Sixth International School: Mathematical Theory in Fluid Mechanics, 2000, Mathematical Theory in Fluid Mechanics.

[3] S.A. Nazarov. On the two-dimensional aperture problem for Navier-Stokes equations. C. R. Acad. Sci. Paris, Ser. 1, 323, $699-703,1996$.

[4] S.A. Nazarov. The Navier-Stokes problem in a two-dimensional domains with angular outlets to infinity. Zapiski Nauchn. Seminarov POMI, 257, 207 - 227, 1999.

[5] S.A. Nazarov and K. Pileckas. Asymptotic conditions at infinity for the Stokes and Navier-Stokes problems in domains with cylindrical outlets to infinity. volume 4 of Advances in Fluid Dynamics, Quaderni di Matematica, 141 - 243, 1999.

[6] S.A. Nazarov and K. Pileckas. Asymptotics of solutions to the Navier-Stokes equations in the exterior of a bounded body. Doklady RAN, 367(4), 461 - 463, 1999.

[7] S.A. Nazarov and K. Pileckas. On steady Stokes and Navier-Stokes problems with zero velocity at infinity in a three-dimensional exterior domain. J. Math. Kyoto Univ., 40(3), 475 - 492, 2000 .

[8] S.A. Nazarov and B.A. Plamenevskii. Elliptic boundary value problems in domains with piecewise smooth boundaries. Walter de Gruyter and Co, Berlin, 1994.

[9] S.A. Nazarov, A. Sequeira and J.H. Videman. Asymptotic behaviour at infinity of threedimensional steady viscoelastic flows. Pacific J. Math. Fluid Mech. (submitted)

[10] S.A. Nazarov, M. Specovius-Nengebaner and G. Thäter. Quiet flows for Stokes and Navier-Stokes problems in domains with cylindrical outlets to infinity. Kyushu J. Math., 53, $369-394,1999$.

[11] A. Novotny and M. Padula. Physically reasonable solutions to steady compressible Navier-Stokes equations in 3D-exterior domains I $\left(v_{\infty}=0\right)$. J. Math. Kyoto Univ., 36(2), $389-423,1996$.

[12] A. Novotny and K. Pileckas. Steady compressible Navier-Stokes equations with large potential forces via a method of decomposition. Math. Meth. in Appl. Sci., 21, 665 $684,1998$.

\section{Spūdaus skysčio tekẻjimo matematinis modelis}

\section{T. Leonavičienè, K. Pileckas}

Šiame darbe išnagrinètas spūdaus skysčio tekèjimo išorinèje srityje $\Omega \subset \mathbb{R}^{3}$ matematinis modelis. Šios problemos sprendimui pritaikyta dekompozicijos schema, kuri leidžia netiesinị uždavinį suskaidyti ị tris paprastesnius tiesinius uždavinius: Neimano tipo, modifikuotą Stokso ir transporto. Šių tiesinių uždavinių sprendinių ieškota specialiose svorinèse funkciju erdvèse su atskirta asimptotika.

Suformuluotos teoremos apie minètų tiesinių ir netiesinio uždavinių sprendinio egzistenciją ir vienatị. Pateikti įrodymų pagrindiniai žingsniai. 